Beaming electromagnetic (or heat-flux) instabilities from the interplay with the electron temperature anisotropies

S. M. Shaaban, M. Lazar, P. H. Yoon, and S. Poedts

Citation: Physics of Plasmas 25, 082105 (2018); doi: 10.1063/1.5042481

View online: https://doi.org/10.1063/1.5042481

View Table of Contents: http://aip.scitation.org/toc/php/25/8

Published by the American Institute of Physics

\section{PHYSICS TODAY}

MANACER'S GUIDE

WHITEPAPERS
READ NOW

PRESENTED BY Accelerate R\&D with
Multiphysics Simulation 


\title{
Beaming electromagnetic (or heat-flux) instabilities from the interplay with the electron temperature anisotropies
}

\author{
S. M. Shaaban, ${ }^{1,2, a)}$ M. Lazar, ${ }^{1,3}$ P. H. Yoon, ${ }^{4,5}$ and S. Poedts ${ }^{1}$ \\ ${ }^{1}$ Centre for Mathematical Plasma-Astrophysics, KU Leuven, Celestijnenlaan 200B, 3001 Leuven, Belgium \\ ${ }^{2}$ Theoretical Physics Research Group, Physics Department, Faculty of Science, Mansoura University, \\ 35516 Mansoura, Egypt \\ ${ }^{3}$ Institut für Theoretische Physik, Lehrstuhl IV: Weltraum- und Astrophysik, Ruhr-Universität Bochum, \\ D-44780 Bochum, Germany \\ ${ }^{4}$ Institute for Physical Science and Technology, University of Maryland, College Park, Maryland 20742, USA \\ ${ }^{5}$ Korea Astronomy and Space Science Institute, Daejeon 34055, Korea School of Space Research, \\ Kyung Hee University, Yongin, Gyeonggi 17104, South Korea
}

(Received 1 June 2018; accepted 13 July 2018; published online 2 August 2018)

\begin{abstract}
In space plasmas, kinetic instabilities are driven by the beaming (drifting) components and/or the temperature anisotropy of charged particles. The heat-flux instabilities are known in the literature as electromagnetic modes destabilized by the electron beams (or strahls) aligned to the interplanetary magnetic field. A new kinetic approach is proposed here in order to provide a realistic characterization of heat-flux instabilities under the influence of electrons with temperature anisotropy. Numerical analysis is based on the kinetic Vlasov-Maxwell theory for two electron counterstreaming (core and beam) populations with temperature anisotropies and stationary, isotropic protons. The main properties of electromagnetic heat-flux instabilities are found to be markedly changed by the temperature anisotropy of the electron beam $A_{b}=T_{\perp} / T_{\|} \neq 1$, leading to stimulation of either the whistler branch if $A_{b}>1$ or the firehose branch for $A_{b}<1$. For a high temperature anisotropy, whistlers switch from heat-flux to a standard regime, when their instability is inhibited by the beam. Published by AIP Publishing. https://doi.org/10.1063/1.5042481
\end{abstract}

\section{INTRODUCTION}

Collision-poor plasmas from space are highly susceptible to the instabilities driven by the kinetic anisotropies of plasma particles. Thus, the electron strahls, or beaming populations, which carry the electron heat-flux in the solar wind, are often associated with enhanced electromagnetic (EM) fluctuations (Lacombe et al., 2014; Lengyel-Frey et al., 1996; and Lin et al., 1998) presumably attributed to the socalled heat-flux instabilities (Gary et al., 1975). Contrary to a magnetic focusing predicted by the theory, the observations show that strahls lose intensity and become wider with heliospheric distance (Maksimovic et al., 2005 and Štverák et al., 2009). In the absence of collisions between particles only the self-generated instabilities can be responsible for this degradation (Gary and Saito, 2007; Pagel et al., 2007; Saito and Gary, 2007; and Vocks et al., 2005). These evidences explain the increasing interest for the heat-flux instabilities (Saeed et al., 2017a,b and Shaaban et al., 2018), and understanding their role in this context implies a detailed examination in conditions specific to solar wind.

The heat-flux instabilities are highly conditioned by the electron beam, and, depending on the relative beam velocity, two distinct branches can be destabilized. Whistlers with a right-handed $(\mathrm{RH})$ circular polarization (in the direction of the uniform magnetic field) are excited by a less energetic beam with velocity lower than thermal speed. Growth rates of the whistler heat flux instability (WHFI) show a non-

\footnotetext{
${ }^{\text {a)} E l e c t r o n i c ~ m a i l: ~ s h a a b a n . m o h a m m e d @ k u l e u v e n . b e ~}$
}

uniform variation, increasing and then decreasing with increasing the beaming velocity (Gary, 1985 and Shaaban et al., 2018). In the second branch, the left handed (LH) firehose heat flux instability (FHFI) (Gary, 1985) is excited by a more energetic beam, with growth rates increasing monotonically with increasing the beam velocity (Gary, 1985; Saeed et al., 2017b; and Shaaban et al., 2018). Recently, Shaaban et al. (2018) have derived the beam velocity thresholds for each of these two instabilities in the absence of temperature anisotropy and described the intermediary regime of transition, where both heat-flux instabilities may co-exist and compete to each other. It has also been shown that effective (counter-) beaming anisotropy is reduced by the suprathermal electrons present in space plasmas, which implies stimulation of the unstable whistlers but inhibition of the firehose instability (FHI).

Beams or counter-beaming populations of electrons are ubiquitous in space plasmas, e.g., during fast winds and coronal mass ejections, and their kinetic implications cannot be isolated from the effects of temperature anisotropies, if both these two sources of free energy are present (Štverák et al., 2008 and Viñas et al., 2010). Here, we indeed show that all known properties of the heat-flux and temperature anisotropy-driven instabilities may be significantly altered by the interplay of beaming electrons and their temperature anisotropy, i.e., $T_{\perp} \neq T_{\|}$. In fact, in such a complex (but realistic) scenario, we deal with two distinct triggers of the same unstable modes. The heat-flux instabilities described above may interplay with the common whistler instability (WI) driven by anisotropic electrons with $T_{\perp}>T_{\|}$(Gary and 
Wang, 1996; Lazar et al., 2018; and Štverák et al., 2008), or the well-known firehose instability (FHI) excited by the electrons with an opposite anisotropy $T_{\perp}<T_{\|}$(Lazar et al., 2018; Paesold and Benz, 1999; and Štverák et al., 2008). Recent studies have investigated these regimes for low-beta $(\beta \leq 0.4)$ electrons and found that WI is inhibited by the beam (growth rates decrease with increasing the beam velocity), while FHFI is insensitive to a temperature anisotropy $T_{\perp}>T_{\|}$of the beam (Saeed et al., 2017b). In an attempt to make a reliable distinction between the heat-flux and temperature anisotropy instabilities, our present study provides an extended comparative analysis, including the solar wind high-beta $(\beta \geq 1)$ conditions, where kinetic instabilities are expected to be more operative. Suprathermal populations are not considered in the present analysis, with the express intention to isolate and describe only the instabilities resulting from the cumulative effects of electron beams and temperature anisotropy.

In Sec. II, we describe the distribution models for the electrons and protons and derive the general dispersion relation for the EM modes, which incorporates the instability cumulative effects of anisotropic electrons. Whistlers are studied in Sec. III and firehose instability in Sec. IV, and then in Sec. V we provide a comparative study of the instability threshold conditions for different regimes, e.g., WHFI, FHFI, WI, and FHI, as resulting from the interplay of electron beam and temperature anisotropy. Section VI summarizes the results obtained in this work with discussions and conclusions.

\section{DISPERSION RELATIONS}

We consider a collisionless quasi-neutral electron-proton plasma with two populations of electrons, namely, the core (subscript $a=c$ ) and the beam (subscript $a=b$ ), counterstreaming in the protons' frame

$$
f_{e}\left(v_{\perp}, v_{\|}\right)=\eta f_{c}\left(v_{\perp}, v_{\|}\right)+\delta f_{b}\left(v_{\perp}, v_{\|}\right),
$$

where $\eta=n_{c} / n_{e}$ and $\delta=1-\eta$ are the relative number densities satisfying neutrality of charge of the electrons (subscript $e$ ) and protons (subscript $p$ ), $n_{e}=n_{c}+n_{b}=n_{p}$. Each component is a drifting bi-Maxwellian

$$
f_{a}\left(v_{\perp}, v_{\|}\right)=\frac{\pi^{-3 / 2}}{\alpha_{a, \perp}^{2} \alpha_{a, \|}} \exp \left[-\frac{v_{\perp}^{2}}{\alpha_{a, \perp}^{2}}-\frac{\left(v_{\|}-U_{a}\right)^{2}}{\alpha_{a, \|}^{2}}\right],
$$

where drifting velocities $U_{a}$ are directed along the magnetic field and satisfy a zero net current condition $n_{c} U_{c}+n_{b} U_{b}$ $=0$. Thermal velocities $\alpha_{a, \|}=\sqrt{2 k_{B} T_{a, \|} / m_{a}}$ and $\alpha_{a, \perp}$ $=\sqrt{2 k_{B} T_{a, \perp} / m_{a}}$ are defined in terms of the anisotropic temperature components, parallel $\left(T_{\|}\right)$and perpendicular $\left(T_{\perp}\right)$ to the ambient magnetic field $\boldsymbol{B}$. If protons are bi-Maxwellian, the linear dispersion relations describing the parallel electromagnetic modes read (Gary, 1985)

$$
\frac{c^{2} k^{2}}{\omega^{2}}=1+\sum_{a=e, c, b} \frac{\omega_{p, a}^{2}}{\omega^{2}}\left[\xi_{a} Z\left(\xi_{a}^{ \pm}\right)+\Lambda_{a}\left\{1+\xi_{a}^{ \pm} Z\left(\xi_{a}^{ \pm}\right)\right\}\right],
$$

where $c$ is the speed of light, $\omega$ is the wave frequency, $k$ is the wave number, $\omega_{p, a}^{2}=4 \pi n_{a} e^{2} / m_{a}$ is the plasma frequency, \pm distinguish between the circular right-handed (RH) and lefthanded (LH) polarizations, respectively, $\Lambda_{a}=A_{a}-1$, in terms of temperature anisotropy $A_{a}=T_{a, \perp}, / T_{a, \|}, \quad \xi_{a}=(\omega$ $\left.-k U_{a}\right) /\left(k \alpha_{a, \|}\right)$, and

$$
Z\left(\xi_{a}^{ \pm}\right)=\frac{1}{\pi^{1 / 2}} \int_{-\infty}^{\infty} \frac{\exp \left(-x^{2}\right)}{x-\xi_{a}^{ \pm}} d t, \quad \Im\left(\xi_{a}^{ \pm}\right)>0
$$

is the plasma dispersion function (Fried and Conte, 1961) of argument

$$
\xi_{a}^{ \pm}=\frac{\omega \pm \Omega_{a}-k U_{a}}{k \alpha_{a, \|}} .
$$

For isotropic protons, we can rewrite (3)

$$
\begin{aligned}
& \frac{\tilde{w}}{\tilde{k} \sqrt{\mu \beta_{p}}} Z\left(\frac{\mu \tilde{w} \pm 1}{\tilde{k} \sqrt{\mu \beta_{p}}}\right)+\eta\left[\Lambda_{c}+\frac{\left(\Lambda_{c}+1\right)\left(\tilde{w}+u_{c} \tilde{k}\right) \mp \Lambda_{c}}{\tilde{k} \sqrt{\beta_{c}}}\right. \\
& \left.\quad \times Z\left(\frac{\tilde{w} \mp 1+u_{c} \tilde{k}}{\tilde{k} \sqrt{\beta_{c}}}\right)\right]+\delta\left[\Lambda_{b}+\frac{\left(\Lambda_{b}+1\right)\left(\tilde{w}-u_{b} \tilde{k}\right) \mp \Lambda_{b}}{\tilde{k} \sqrt{\beta_{b}}}\right. \\
& \left.\quad \times Z\left(\frac{\tilde{w} \mp 1-u_{b} \tilde{k}}{\tilde{k} \sqrt{\beta_{b}}}\right)\right]=\tilde{k}^{2}
\end{aligned}
$$

in terms of the normalized quantities, $\tilde{k}=k c / \omega_{p, e}, \tilde{w}=\omega /$ $\left|\Omega_{e}\right|$, the proton-electron mass ratio $\mu=m_{p} / m_{e}$, the plasma beta for the population of sort $a, \beta_{a}=8 \pi n_{a} k_{B} T_{a, \|} / \boldsymbol{B}^{2}$, and relative velocities of the beam and core components, $u_{b}=U_{b} \omega_{p, e} /\left(c \Omega_{e}\right)$ and $u_{c}=\delta u_{b} /(1-\delta)$, respectively.

Plasma parameters used in the numerical calculations are given in Table I, unless otherwise specified. These parameters are inspired from the solar wind observations providing electron data from different heliocentric distances. Relevant are the electron data making distinction between core and beaming (strahl) components, see the density contrasts in Štverák et al. (2009) (Figs. 4 and 8), which suggest an average (representative) value $\delta=n_{b} / n_{0}=0.05$. Presuming that strahl and halo electrons comprise the main source of the heat flux transport in the solar wind, for the beam-core temperature contrast the observations estimate a variation between 3 and 13 along the heliocentric distance with an increasing tendency during the fast winds (Pierrard et al., 2016; Pilipp et al., 1987; and Viñas et al., 2010). In this case, we assume $T_{b, \|} / T_{c, \|}=10$. Seeking generalization, for the temperature anisotropy, which is a key parameter in this study, we adopt moderate values typically reported in the solar wind (Phillips et al., 1989; Pierrard et al., 2016;

TABLE I. Parameters for the $j$-component of electrons.

\begin{tabular}{lccc}
\hline \hline & Beam electrons $(h)$ & Core electrons $(c)$ & Ions $(i)$ \\
\hline$n_{j} / n_{i}$ & 0.05 & 0.95 & 1.0 \\
$T_{j, \|} / T_{i, \|}$ & 10.0 & 1.0 & 1.0 \\
$m_{j} / m_{i}$ & $1 / 1836$ & $1 / 1836$ & 1.0 \\
$T_{j, \perp} / T_{j, \|}$ & $\neq 1.0$ & $\neq 1.0$ & 1.0 \\
\hline \hline
\end{tabular}


(a) $\delta=0.05, \beta_{\mathrm{c}}=0.04, u_{b}=0.6$

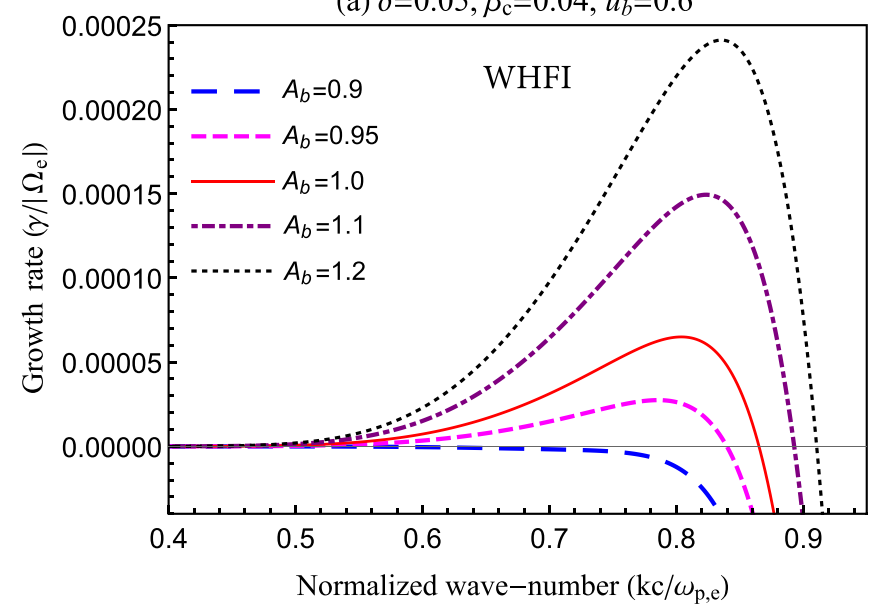

(b) $\delta=0.05, \beta_{\mathrm{c}}=0.04, u_{b}=0.6$

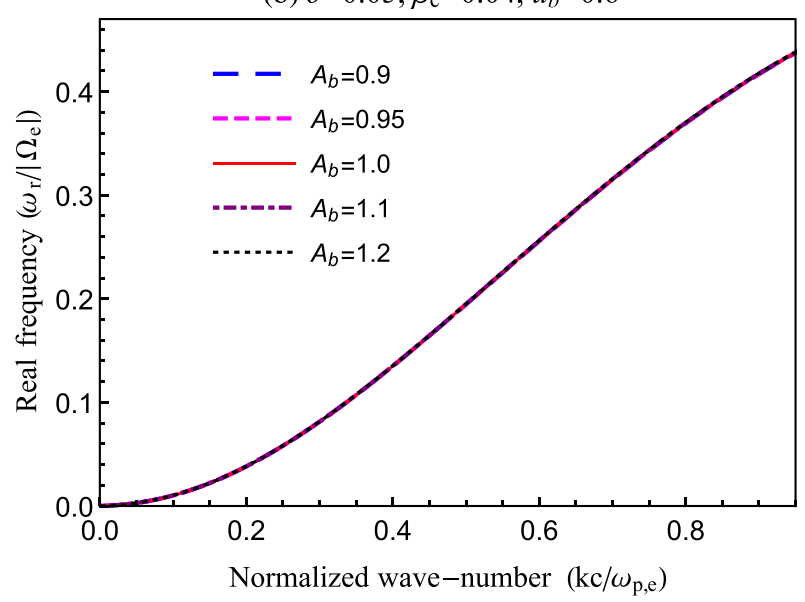

FIG. 1. WHFI: Effects of the beam anisotropy $A_{b}$ on the growth rates (panel a) and wave frequencies (panel b). The plasma parameters are mentioned in each panel velocity.

Pilipp et al., 1987; and Štverák et al., 2008). For the beam velocity values are chosen to ensure conditions for both the WHFI and FHFI, which may also be relevant for the beaming electrons in space plasmas (Pulupa et al., 2014).

\section{UNSTABLE WHISTLER MODES}

We start the analysis with the dispersive characteristics of the whistler modes driven unstable by the interplay of the beam-core counter-streaming electrons and their temperature anisotropies. These are solutions of the dispersion relation (3) for the RH modes with $\xi_{p}^{+}$. The less energetic beams are susceptible to the whistler heat flux instability (WHFI) (Shaaban et al., 2018), which is examined in Figs. 1 and 2 for the following plasma parameters $\delta=0.05, \beta_{c}=\beta_{p}=0.04$, $u_{b}=0.6$. In Fig. 1 , we isolate the effects of the beam anisotropy by considering isotropic core with $A_{c}=1.0$ and show the influence of the beam anisotropy $A_{b}=0.9,0.95,1.0$, $1.1,1.2$ on the growth rates (panel a) and wave-frequencies (panel b) of WHFI. Growth rates are markedly enhanced by increasing the temperature anisotropy in perpendicular direction, $A_{b}>1$, and are inhibited by an opposite anisotropy in parallel direction, $A_{b}<1$. The corresponding wave- (a) $\delta=0.05, \beta_{\mathrm{c}}=0.04, u_{b}=0.6$

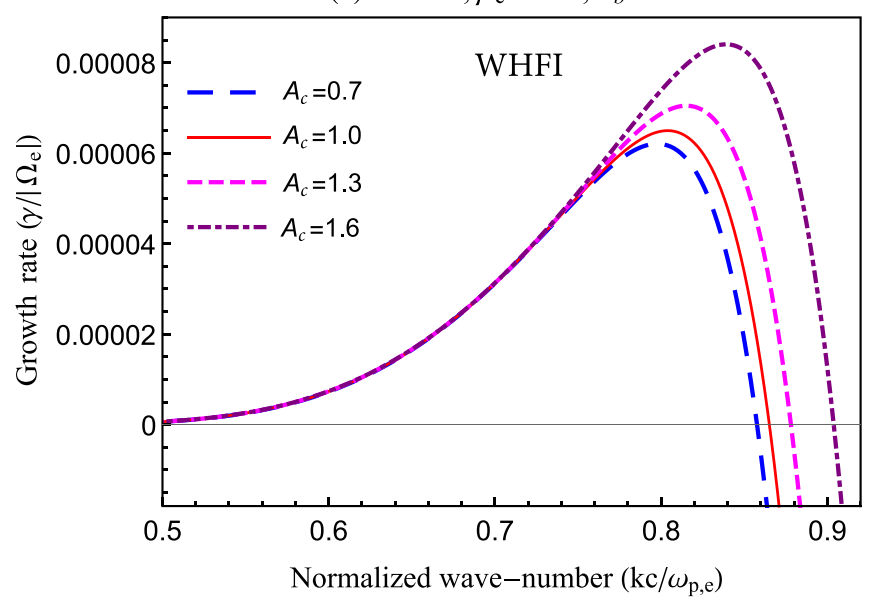

(b) $\delta=0.05, \beta_{\mathrm{c}}=0.04, u_{b}=0.6$

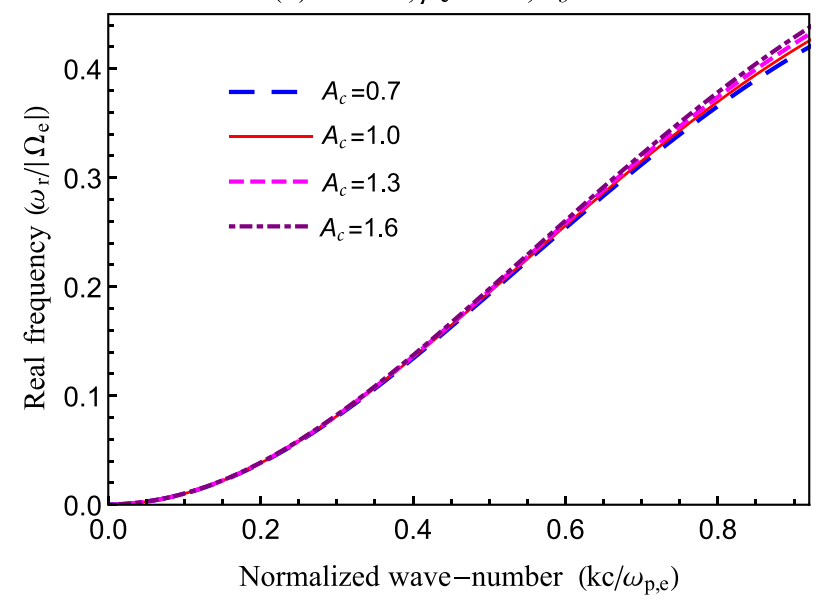

FIG. 2. WHFI: Effects of the core anisotropy $A_{c}$ on the growth rates (panel a) and wave frequencies (panel b). The plasma parameters are mentioned in each panel.

frequencies remain unaffected by the variation of the beam temperature anisotropy. These unstable solutions are derived for relatively low anisotropies of the beam $\left(0.9 \leq A_{b} \leq 1.2\right)$, and a low plasma beta of the core $\beta_{c}=0.04$, to avoid the whistler or firehose instability effects driven by the temperature anisotropies. For higher anisotropies of the beam $A_{b}>1.2$, whistlers exhibit significant growth rates characteristic to the whistler instability (WI) driven by the temperature anisotropy, and it becomes difficult to distinguish between the WHF and WI regimes, as discussed later in Fig. 4. In Fig. 2, we assume an isotropic beam $\left(A_{b}=1\right)$ and outline the effect of the core anisotropy $A_{c}=0.7,1.0,1.3,1.6$ on the growth rates (panel a) and wave-frequencies (panel b) of WHFI. The growth rates change only slightly, being enhanced by the core anisotropy in the perpendicular direction $A_{c}>1$, but inhibited by an opposite anisotropy in parallel direction $A_{c}<1$. Clearly, the anisotropic beam has a higher influence on the instability: for $A_{b}=1.1$ maximum growth rate in Fig. 1 is three times higher than that obtained for $A_{c}=1.6$ in Fig. 2. For reference, the growth rates for isotropic isotropic temperatures $A_{b}=A_{c}=1$ are displayed in both figures with red solid lines. 
(a) $\delta=0.05, \beta_{\mathrm{c}}=0.04, \mathrm{~A}_{\mathrm{c}}=1, \mathrm{~A}_{\mathrm{b}}=3$

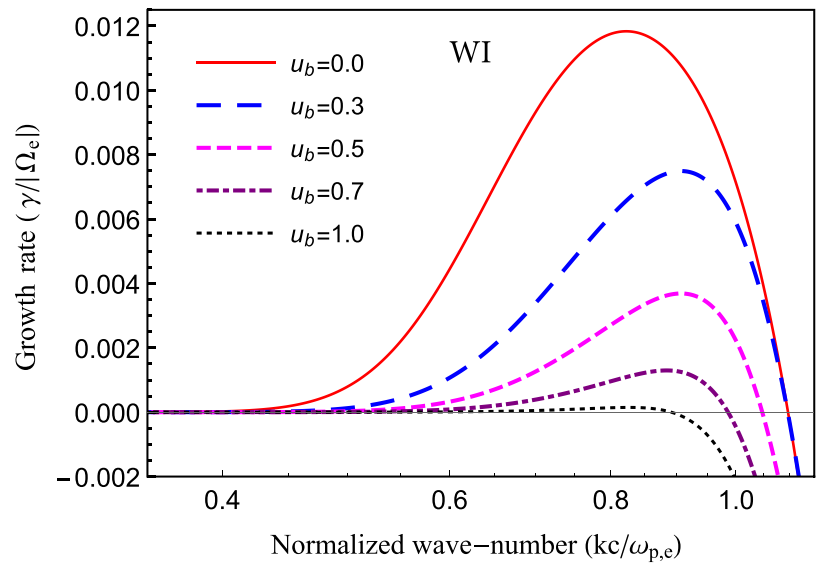

(b) $\delta=0.05, \beta_{\mathrm{c}}=0.04, \mathrm{~A}_{\mathrm{c}}=3, \mathrm{~A}_{\mathrm{b}}=1$

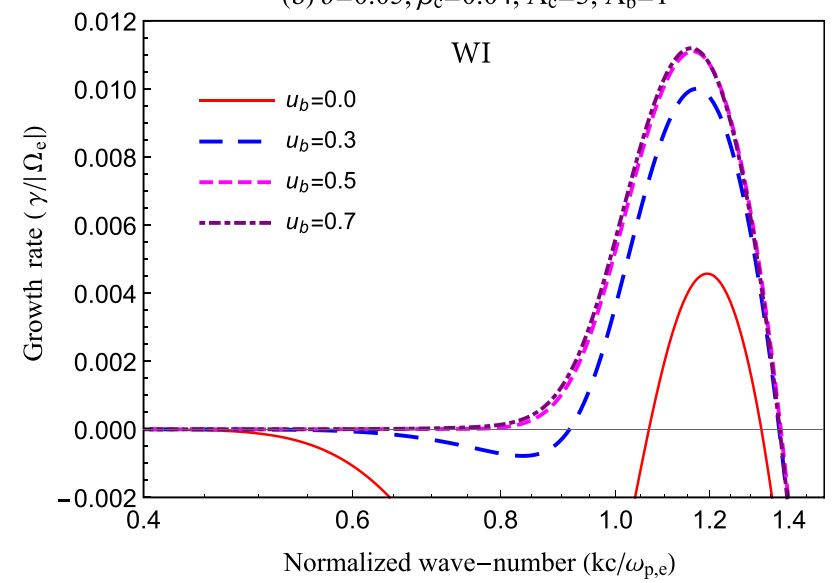

FIG. 3. WI: Effects of the beam velocity $u_{b}$ on the growth rates of the WI driven either by an anisotropic beam $A_{b}=3.0$ (panel a) or by an anisotropic core $A_{c}=3.0$ (panel b). The other plasma parameters are mentioned in each panel.

The temperature anisotropy driven instabilities are usually studied in the absence of beaming components. Figure 3 shows the effect of beaming velocity $u_{b}$ on the WI driven by a higher temperature anisotropy, for $\delta=0.05$ and $\beta_{c}=\beta_{p}=0.04$. When WI is driven by anisotropic beam with $A_{b}=3.0$ (panel a), the effective anisotropy diminishes with increasing the beam speed $u_{b}$ and the instability is inhibited, reducing growth rates and the intervals of unstable wave-numbers. By contrast, growth rates driven by the anisotropic core with $A_{c}=3.0$ (panel b) are enhanced by increasing $u_{b}$ and saturate for $u_{b} \geq 0.5$, resembling a regime characteristic to WHFI. The wave frequency (not shown here) only slightly decreases by increasing $u_{b}$. WHFI has dispersive characteristics similar to WI. Both instabilities are driven by resonant electrons and display maximum growth rates in directions parallel to the background magnetic field (a) $\delta=0.05, \beta_{\mathrm{c}}=0.04, \mathrm{~A}_{\mathrm{b}}=\mathrm{A}_{\mathrm{c}}=1.0$

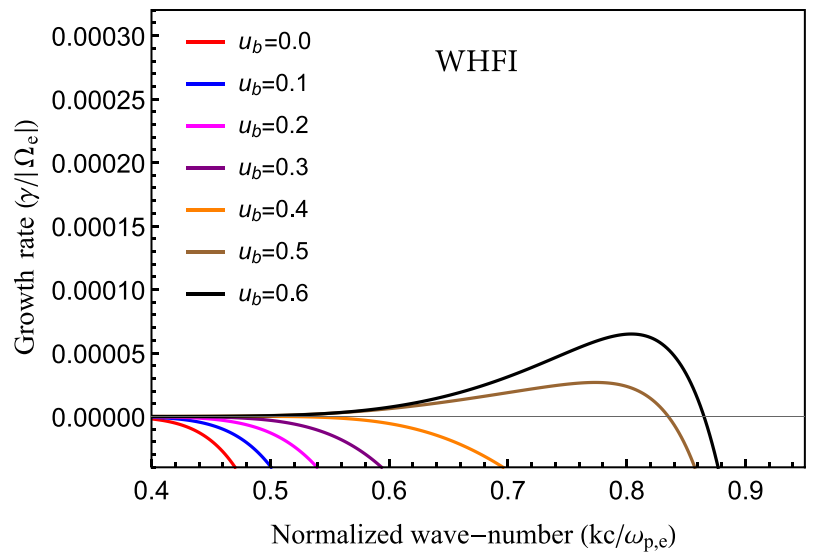

(c) $\delta=0.05, \beta_{\mathrm{c}}=0.04, \mathrm{~A}_{\mathrm{b}}=1.5, \mathrm{~A}_{\mathrm{c}}=1$

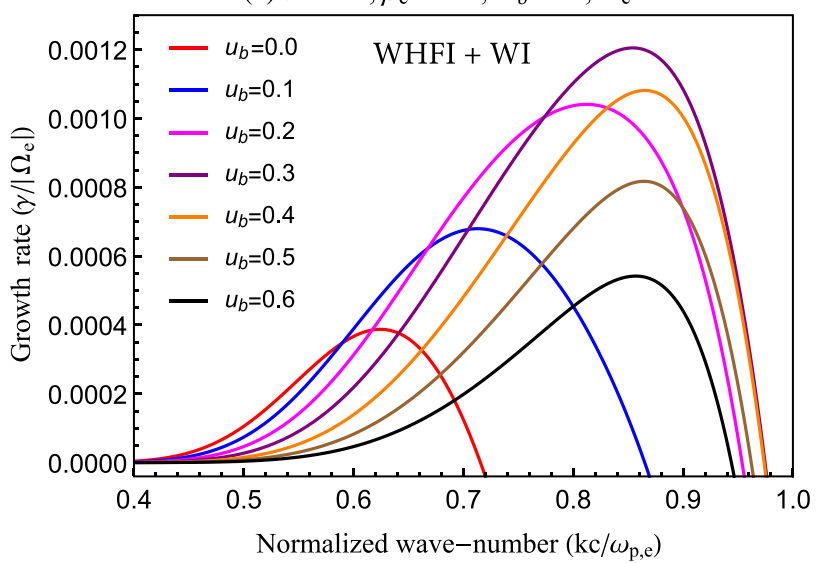

(b) $\delta=0.05, \beta_{\mathrm{c}}=0.04, \mathrm{~A}_{\mathrm{b}}=1.2, \mathrm{~A}_{\mathrm{c}}=1$

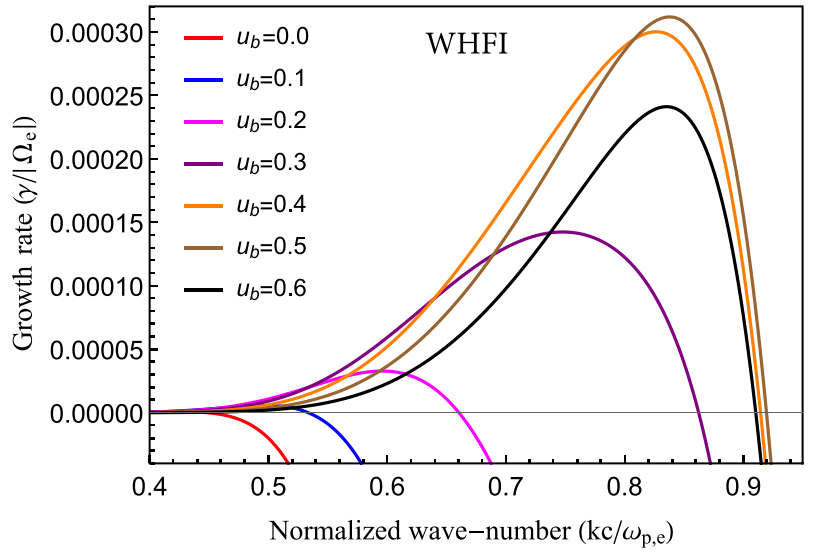

(d) $\delta=0.05, \beta_{\mathrm{c}}=0.04, \mathrm{~A}_{\mathrm{b}}=2.5, \mathrm{~A}_{\mathrm{c}}=1$

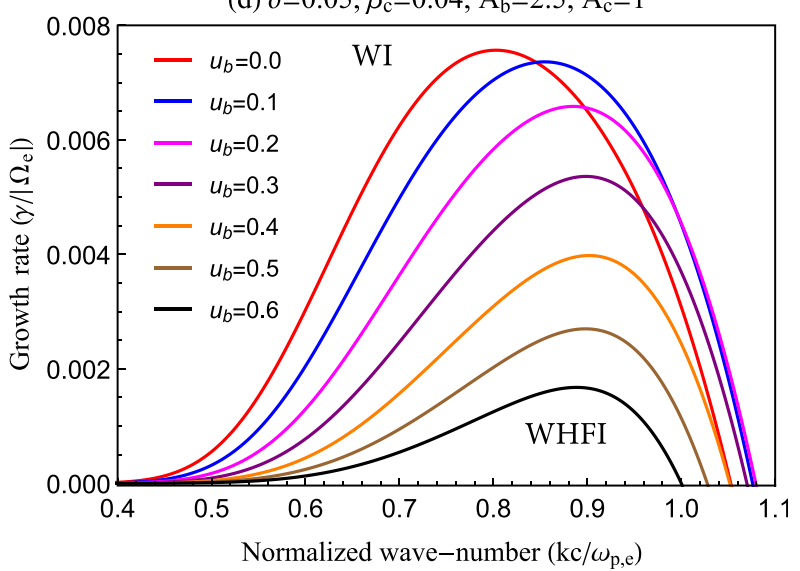

FIG. 4. Effect of the of the beam velocity $u_{b}$ on the cumulative whistler instabilities for beam anisotropies $A_{b}=1.0$ (panel a), $A_{b}=1.2$ (panel b), $A_{b}=1.5$ (panel c), and $A_{b}=2.5$ (panel d). The plasma parameters are mentioned in each panel. 
(a) $\delta=0.05, \beta_{\mathrm{c}}=1.2, \mathrm{~A}_{\mathrm{c}}=1, u_{b}=3.8$

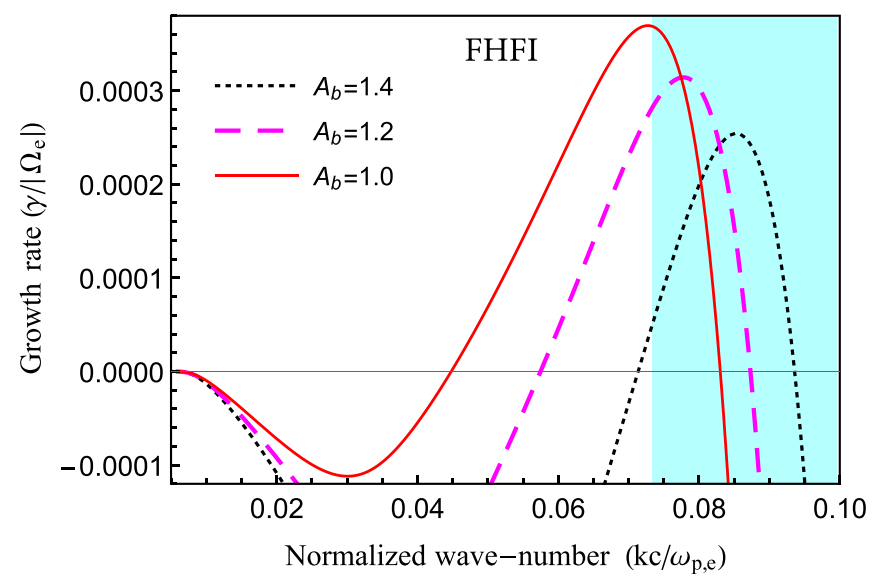

(b) $\delta=0.05, \beta_{\mathrm{c}}=1.2, \mathrm{~A}_{\mathrm{c}}=1, u_{b}=3.8$

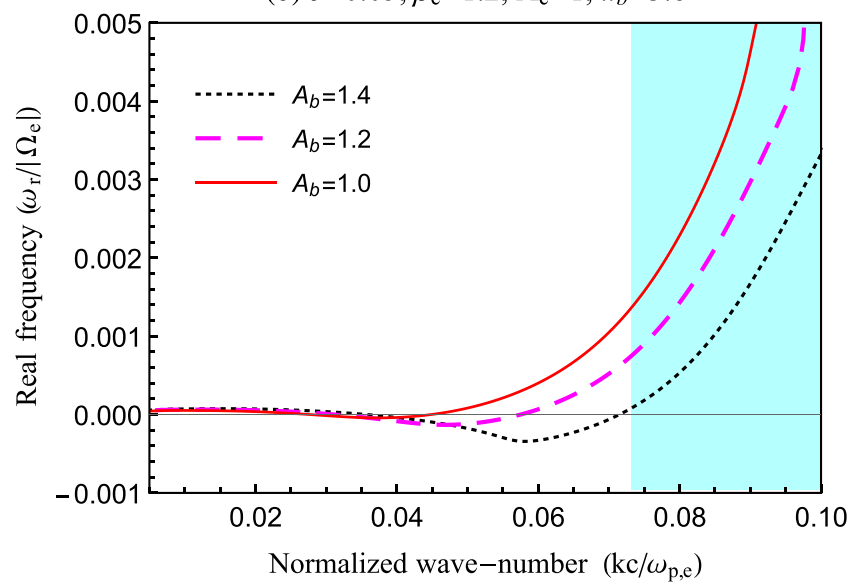

FIG. 5. FHF: Effect of beam anisotropy $A_{b}>1$ on the growth rates (panel a) and wave frequency (panel b).

(when the modes are right-hand circularly polarized) (Gary, 1993 and Lazar et al., 2018). However, WHFI and WI represent two distinct regimes of whistler modes, destabilized by, respectively, the beam $u_{b}$ and temperature anisotropy $A_{b}>1$.

Figure 4 presents four distinct regimes of destabilized whistler modes, assuming $\delta=0.05, A_{c}=1.0$, and $\beta_{c}=\beta_{p}$ $=0.04$. In panel (a), we consider, for reference, the beam isotropic $A_{b}=1.0$ and find growth or damping rates of whistlers varying only under the influence of the beam velocity $u_{b}$ : the instability is obtained for higher beaming velocities $u_{b}=0.5,0.6$, and maximum growth rates are obtained for $u_{b}=0.6$ (Shaaban et al., 2018). In panel (b), for a relatively small beam anisotropy $A_{b}=1.2$, growth rates are markedly stimulated by the beam velocity, and maximum growth rates are obtained for a less energetic beam, i.e., $u_{b}=0.5$ (brown line). Higher anisotropies $A_{b}=1.5,2.5$ may drive an instability with significantly high growth rates (even in the absence of a beam, $u_{b}=0$ ), see red lines in panels (c) and (d). In panel (c), the instability features characteristics of both the WHFI and WI, cumulating the effects of beam and temperature anisotropy. Maximum growth rates are obtained for $u_{b}=0.3$. For higher anisotropies $A_{b}=2.5$, in panel (d), (a) $\delta=0.05, \beta_{\mathrm{c}}=1.2, \mathrm{~A}_{\mathrm{c}}=1, u_{b}=3.8$

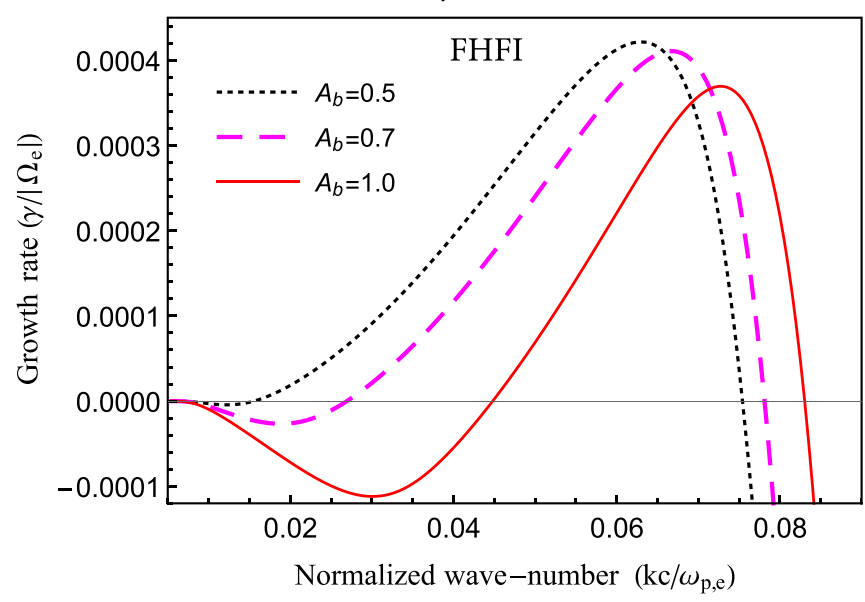

(b) $\delta=0.05, \beta_{\mathrm{c}}=1.2, \mathrm{~A}_{\mathrm{c}}=1, u_{b}=3.8$

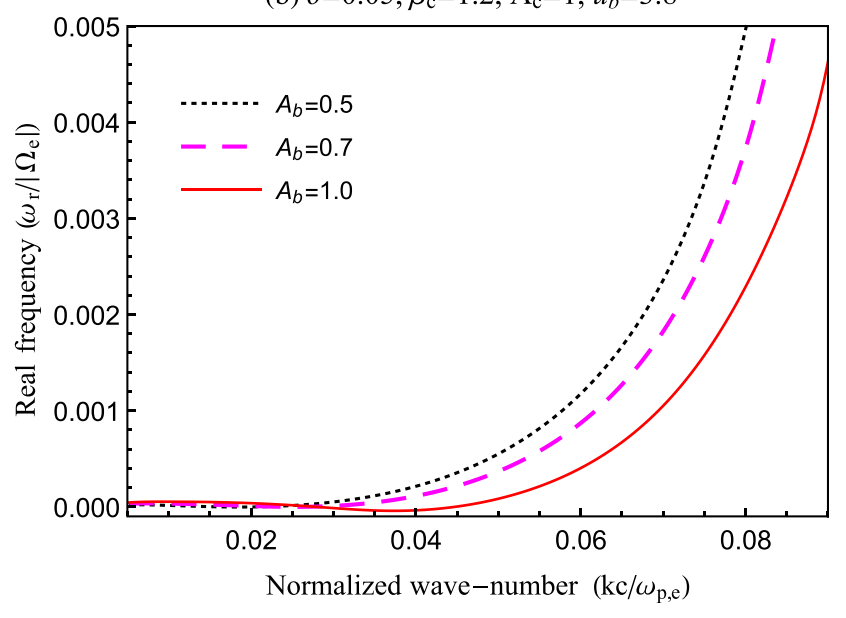

FIG. 6. FHF: The same in Fig. 5 but for $A_{b}<1.0$.

we obtain WI-like growth rates (maximum for $u_{b}=0$ ) which decrease as the beam velocity increases. More energetic beams, e.g., $u_{b}=0.6$ (black line), may determine another transition to WHFI regime.

A series of conclusions can already be drawn, which enable to distinguish between these two regimes of unstable whistlers (also see Sec. V of the instability thresholds). Thus, the beam anisotropy $A_{b}>1.0$ stimulates the WHFI, reducing also the beam velocity required for the instability to display maximum growth rate. On the other hand, growth rates of WI are reduced by the beam, and an increase in $u_{b}$ may trigger a transition to the WHFI.

\section{UNSTABLE ELECTRON FIREHOSE MODES}

In this section, we investigate the LH branch of HFIs represented by the electron firehose heat-flux instability (FHFI). Conditions of this instability are expected to be markedly modified under the influence of temperature anisotropies $A_{b, c}<1$, which are responsible for the excitation of standard firehose instability (FI). For sufficiently large core plasma beta $\beta_{c}$ and high beaming velocity $u_{b}>2.7$, both the FHFI and FI are expected to develop with similar dispersive features (Gary, 1993 and Shaaban et al., 2018). First we 
(a) $\delta=0.05, \beta_{\mathrm{b}}=0.36, \beta_{\mathrm{c}}=0.04, \mathrm{~A}_{\mathrm{c}}=1, \mathrm{~A}_{\mathrm{b}}=3.5$

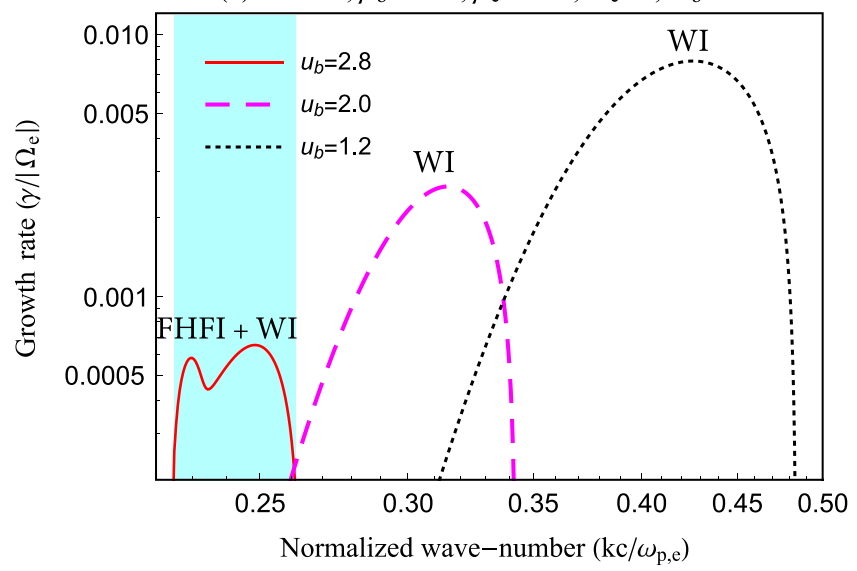

(b) $\delta=0.05, \beta_{\mathrm{b}}=0.36, \beta_{\mathrm{c}}=0.04, \mathrm{~A}_{\mathrm{c}}=1, \mathrm{~A}_{\mathrm{b}}=3.5$

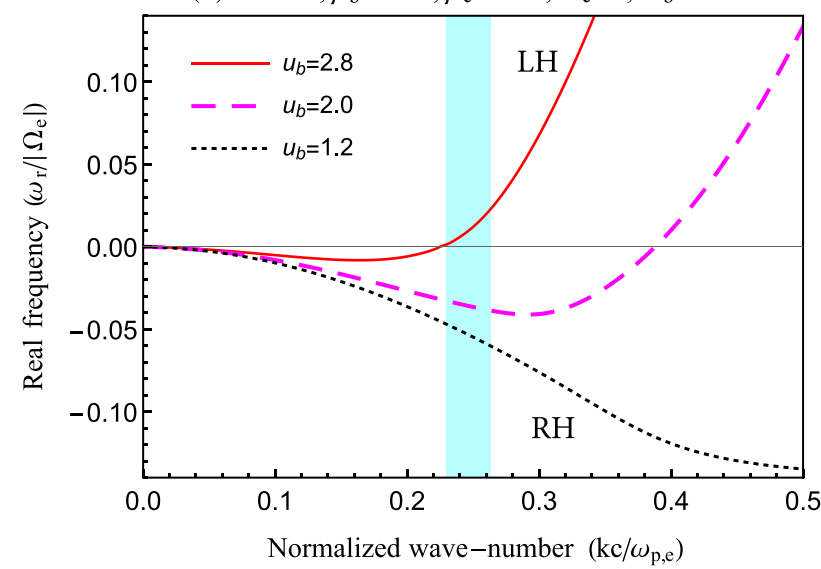

(a) $\delta=0.05, \beta_{\mathrm{b}}=0.36, \beta_{\mathrm{c}}=0.04, \mathrm{~A}_{\mathrm{c}}=1, \mathrm{~A}_{\mathrm{b}}=3.5$

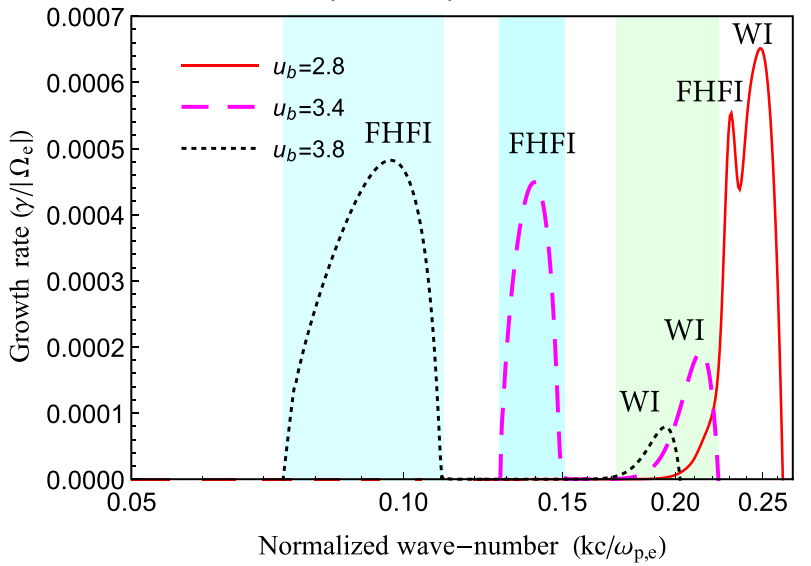

(b) $\delta=0.05, \beta_{\mathrm{b}}=0.36, \beta_{\mathrm{c}}=0.04, \mathrm{~A}_{\mathrm{c}}=1, \mathrm{~A}_{\mathrm{b}}=3.5$

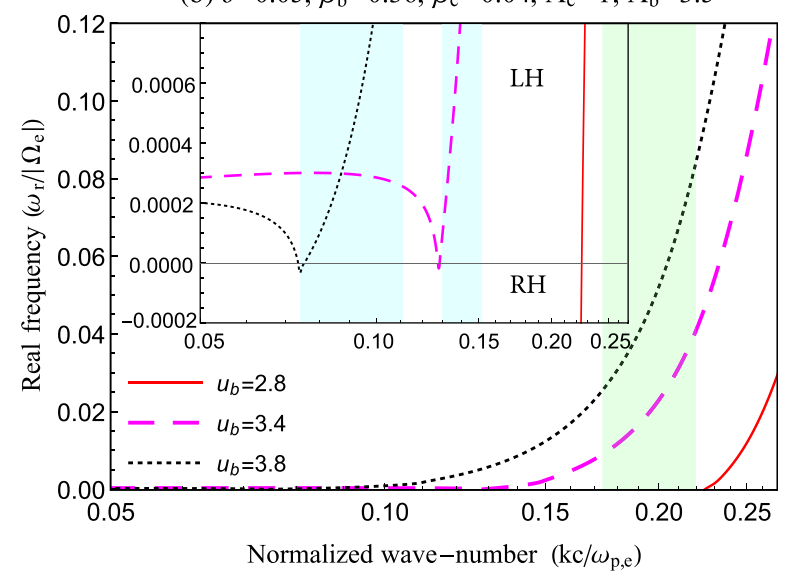

FIG. 7. Effect of the beam velocity $u_{b}$ on the growth rates (panel (a)) and wave frequency (panel b) of the WI instability driven by beam anisotropy $A_{b}=3.5$.

analyze the FHFI under the mutual effects of the electron beam $\left(u_{b} \neq 0\right)$ and its temperature anisotropy $\left(A_{b} \neq 1\right)$. The unstable solutions are obtained by solving numerically the dispersion relation (5) for LH modes with $\xi_{p}^{-}$. By contrast to recent studies of FHFI, which consider only small plasma beta regimes, i.e., $\beta_{c}=0.04$ (Saeed et al., 2017b), here we assume solar wind high beta conditions, i.e., $\beta_{c}=\beta_{p}>1$, which are more favorable to FHIs.

In Figs. 5 and 6, we assume $\delta=0.05, \beta_{c}=\beta_{p}=1.2$, $A_{c}=1.0$, and more energetic beams $u_{b}=3$.8. Figure 5 shows the effects of an increasing anisotropy $A_{b}=1.0,1.2,1.4$ on the FHFI: in panel (a), growth rates decrease and the range of unstable wave numbers increases, and in panel (b), the wave frequency exhibits the same monotonous increasing. An opposite anisotropy $A_{b}=1.0,0.7,0.5$, assumed in Fig. 6, has a cumulative effect stimulating the FHFI by increasing the growth rates and wave-frequencies. The wave frequency keeps the positive sign $\omega_{r}>0$ in the range of the FHF peaks. The core anisotropy $A_{c} \neq 1$ manifests similar effects on the FHFI (not shown here).

In Sec. III, we have outlined a transition from WI to WHFI, triggered by the increase in the beam speed $u_{b}$, when the temperature anisotropy of the beam is relatively small. Here, in Fig. 7, we show that, provided the anisotropy is high enough, i.e., $A_{b}=3.5$, WI can directly convert to FHFI with increasing $u_{b}=1.2,2.0,2.8$. The WI is driven by the beam anisotropy $A_{b}=3.5$ for the same plasma parameters invoked in Saeed et al. (2017b) (their Fig. 3): $\beta_{c}=0.04, \beta_{b}=0.36$, $\delta=0.05$, and $A_{c}=1.0$. Top panels present the first regime where the WI instability is dominant and the beaming velocity $u_{b}<2.8$ is below but close to the threshold value for the excitation of FHFI (Saeed et al., 2017b and Shaaban et al., 2018). Increasing the beam velocity has an inhibiting effect leading to a decrease in both the growth rates and the range of unstable wave numbers of WI (panel a). The corresponding wave frequencies (panel b) decrease and remain RH polarized $\left(\omega_{r}<0\right)$ in the range of the WI instability peaks, unless for energetic beams when the polarization changes to LH (cyan areas) under the influence of FHFI which exhibit a second distinct peak of growth rates (red solid line). The double-peak growth rates are relevant for the transition between the two regimes of FHFI and WI. FHFI becomes dominant for more energetic beams $\left(u_{b}>2.8\right)$, when the FHF peak is markedly enhanced moving towards lower wave-numbers (bottom panels c and d). Small peaks of WI are decoupled and still visible, but remain LH polarized (green area). Such reversals of the whistler mode polarizations have been observed by STEREO in the Earth's inner plasma-sphere at $L<2$ (Breneman et al., 2011).

For a core with a sufficiently large plasma beta, a beam with an excess of parallel temperature, i.e., $A_{b}<1$, may excite the electron firehose $(\mathrm{EFH})$ instability. Figure 8 shows the effect of the beam velocity $u_{b}$ on the EFH instability driven by a 
(a) $\delta=0.05, \beta_{\mathrm{c}}=4.0, \mathrm{~A}_{\mathrm{b}}=0.2, \mathrm{~A}_{\mathrm{c}}=1.0$

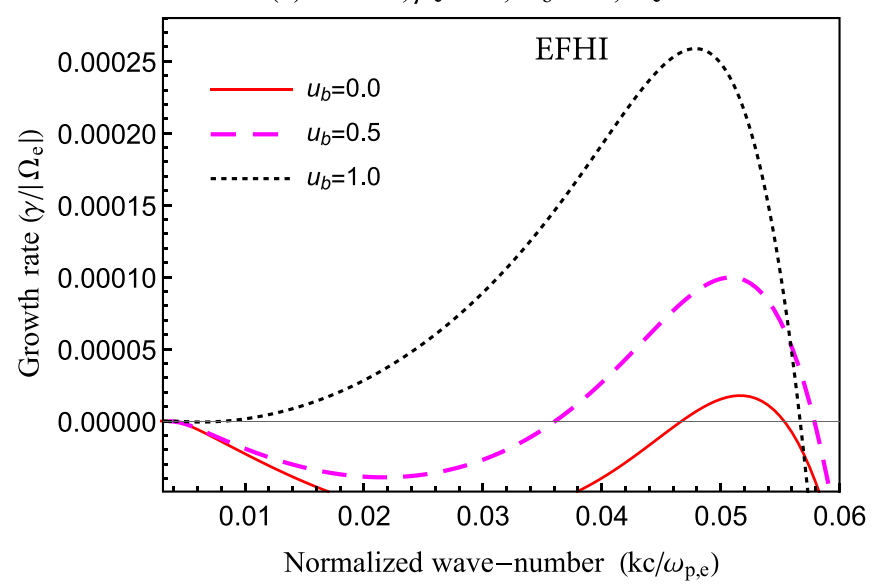

(b) $\delta=0.05, \beta_{\mathrm{c}}=4.0, \mathrm{~A}_{\mathrm{b}}=0.2, \mathrm{~A}_{\mathrm{c}}=1.0$

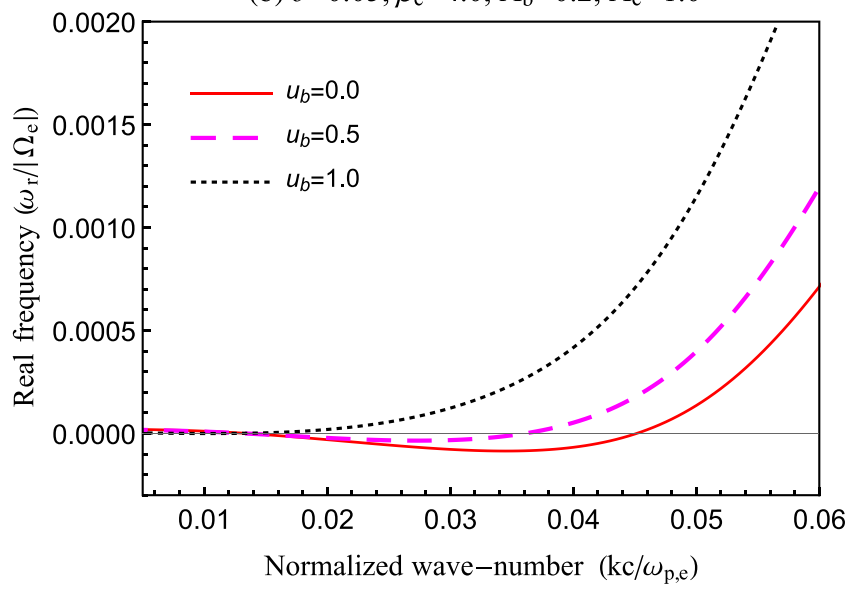

FIG. 8. Effect of the beam velocity $u_{b}$ on the growth rates (panel a) and wave frequency (panel b) of the EFHI driven by beam anisotropy $A_{b}=0.2$.

temperature anisotropy $A_{b}=0.2$ for the following plasma parameters $\delta=0.05, \beta_{c}=\beta_{p}=\beta_{b} / 10=4.0, A_{c}=1.0$. The growth rates (panel a) and wave frequencies (panel b) of EFH instability are significantly stimulated by increasing $u_{b}=0.0$, $0.5,1.0$. These effects contrast with the inhibition of WI shown in Fig. 7.

\section{THRESHOLD CONDITIONS}

Thresholds offer a concise but more comprehensive picture of the unstable regimes. Figures 9-11 present the instability thresholds derived for a small maximum growth rate $\gamma_{m}=2 \times 10^{-4}\left|\Omega_{e}\right|$, approaching marginal stability, i.e., $\gamma_{m} \rightarrow 0$. These thresholds are derived in terms of the instability drivers, i.e., beam velocity $u_{b}$ or temperature anisotropy $A_{b}$, as a function of the core plasma beta $\beta_{c}$. The other plasma parameters are kept constant, e.g., $\delta=0.05$ and $A_{c}=1.0$. Mathematically, the instability thresholds are fitted to a function of $\beta_{c}$ generically expressed by (Shaaban et al., 2016)

$$
\Delta=\left(1+\frac{a}{\beta_{c}^{b}}\right) \frac{c}{\beta_{c}^{d}}
$$

where (a) $\gamma_{\mathrm{m}} /\left|\Omega_{\mathrm{e}}\right|=2 \times 10^{-4}, \delta=0.05, \mathrm{~A}_{\mathrm{c}}=1.0$
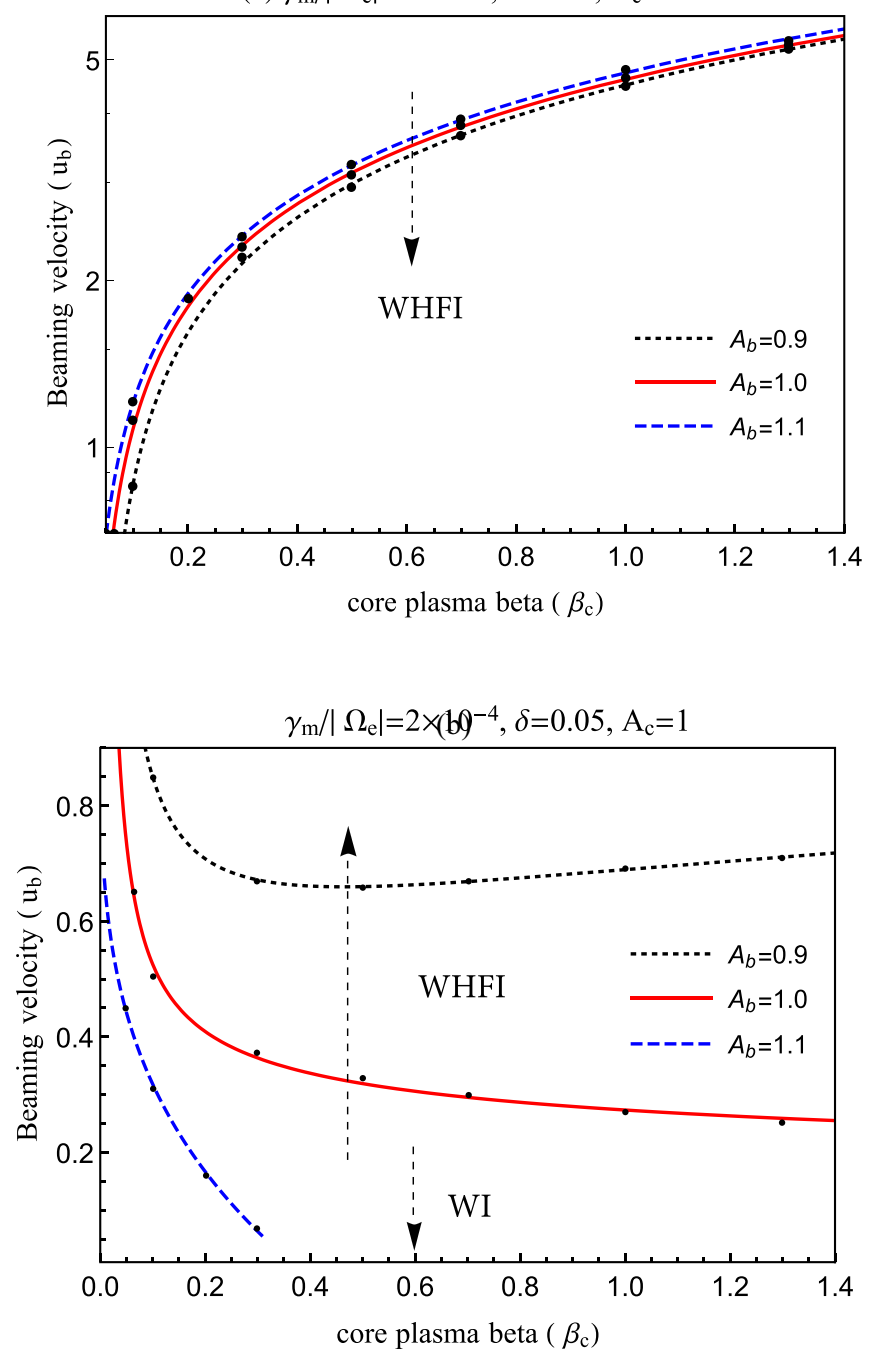

FIG. 9. Effect of the beam anisotropy $A_{b}$ on the (a) upper and (b) lower thresholds $\left(\gamma_{m}=2 \times 10^{-4}\left|\Omega_{e}\right|\right)$ of the WHFI.

$$
\Delta= \begin{cases}A_{b}, & \text { for temperature anisotropy instabilities } \\ u_{b}, & \text { for heatfluxinstabilities. }\end{cases}
$$

Fitting parameters $a, b$, and $c$ are tabulated in Tables II-IV in the Appendix.

As shown in Fig. 4(a), the WHFI growth rates vary nonuniformly with increasing the beaming velocity, suggesting, as also shown recently by Shaaban et al. (2018), that the unstable WHF modes are bounded between two thresholds of the beam velocity. Figure 9 describes the effect of temperature anisotropy $A_{b} \neq 1.0$ on the upper and lower thresholds of WHFI, in panels (a) and (b), respectively. We contrast thresholds for an isotropic beam $\left(A_{b}=1\right.$, solid-red), with those for $A_{b}=1.1$ (dashed-blue) and $A_{b}=0.9$ (dottedblack). In panel (a), the upper threshold is slightly increased by the anisotropy in the perpendicular direction, $A_{b}=1.1$, but it is slightly decreased by an opposite anisotropy in the parallel direction, $A_{b}=0.9$. Only small variations are obtained in this case, given that this regime of WHFI is mainly controlled by relatively high beaming velocities, and temperature anisotropies are relatively small. However, for the same anisotropies, in panel (b) the lower WHFI threshold 
(a) $\gamma_{\mathrm{m}} /\left|\Omega_{\mathrm{e}}\right|=2 \times 10^{-4}, \delta=0.05, \mathrm{~A}_{\mathrm{c}}=1$

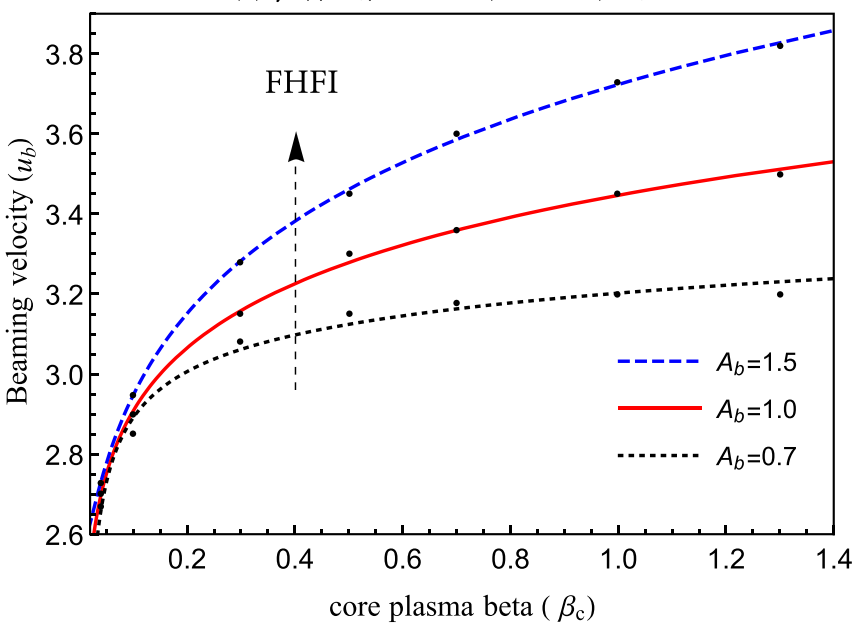

(b) $\gamma_{\mathrm{m}} /\left|\Omega_{\mathrm{e}}\right|=2 \times 10^{-4}, \delta=0.05, \mathrm{~A}_{\mathrm{c}}=1$

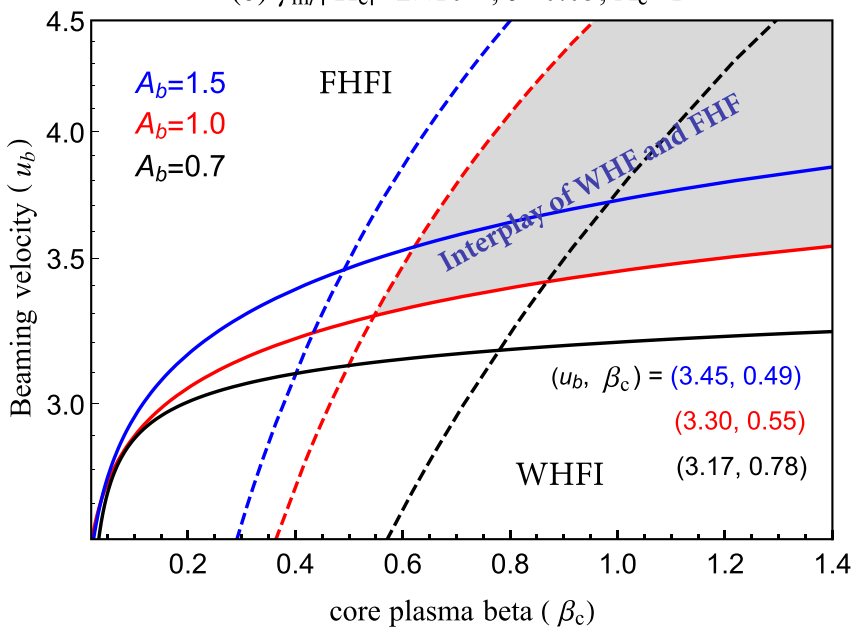

FIG. 10. Effect of the beam anisotropy $A_{b}$ on the (a) FHFI threshold and a comparison with the (b) WHFI upper threshold for $\gamma_{m}=2 \times 10^{-4}\left|\Omega_{e}\right|$.

undergoes more important changes. This threshold is markedly enhanced for $A_{b}=0.9$, and this difference is increased with increasing the core plasma beta $\beta_{c}$, squeezing the unstable regime of WHFI (upper directed arrow). In an opposite situation for $A_{b}=1.1$, the lower WHFI threshold is markedly reduced to lower beaming velocities $u_{b}$, and with increasing $\beta_{c}$ the WI peak starts to dominate the WHFI peak, which finally quenches completely. This regime marks the transition from WHFI to the most common WI (lower directed arrow) which becomes exclusively driven by the temperature anisotropy (for $u_{b}=0$ and $\beta>\beta_{c} \simeq 0.3$ ).

Figure 10(a) describes the effect of temperature anisotropy on the FHFI thresholds, by contrasting thresholds derived for an isotropic beam $\left(A_{b}=1\right.$, solid-red) with those for $A_{b}>1.0$ (dashed-blue) and $A_{b}<1.0$ (dotted-black). Variations in this case resemble those of the WHFI upper threshold, but the most unstable FHF modes are situated above the thresholds, as pointed out by the dashed arrow. Temperature anisotropy $A_{p}=1.5$ has a stimulating effect on the FHFI threshold, squeezing the unstable regime of the FHF modes, while for $A_{b}=0.7$ the threshold is reduced (a) $\gamma_{\mathrm{m}} /\left|\Omega_{\mathrm{e}}\right|=2 \times 10^{-4}, \delta=0.05, \mathrm{~A}_{\mathrm{c}}=1$

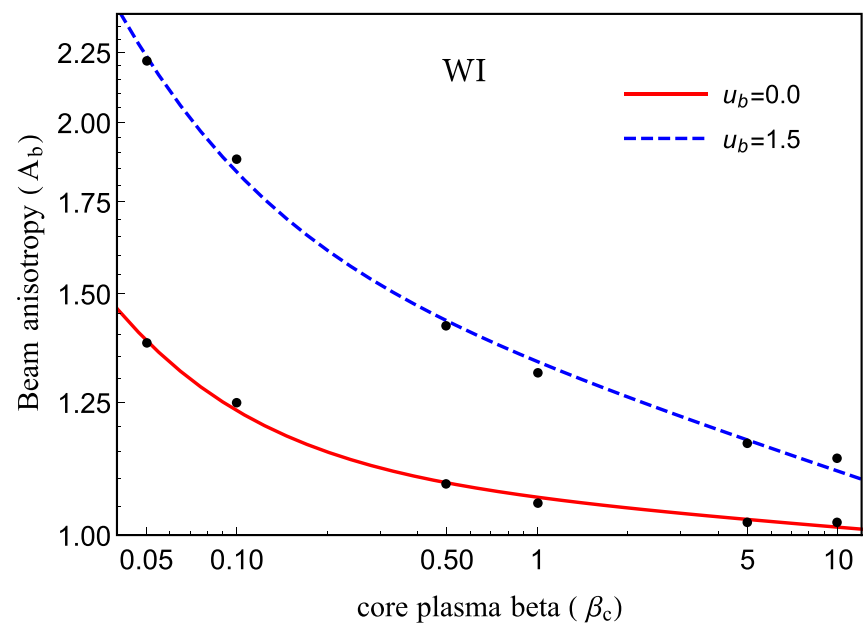

(b) $\gamma_{\mathrm{m}} /\left|\Omega_{\mathrm{e}}\right|=2 \times 10^{-4}, \delta=0.05, \mathrm{~A}_{\mathrm{c}}=1$

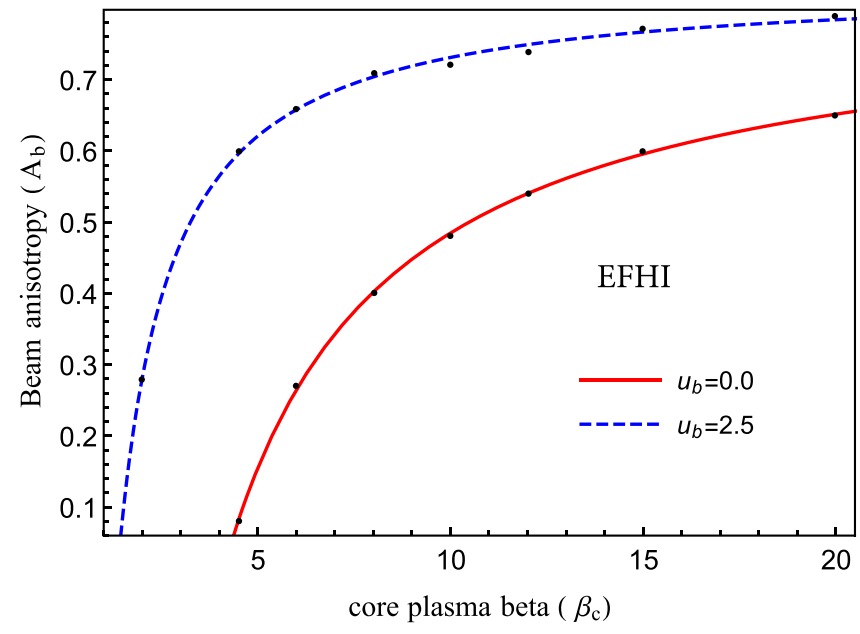

FIG. 11. Effect of the beam velocity $u_{b}$ on WI (panel a) and EFH (panel b) instability thresholds with maximum growth rates $\gamma=2 \times 10^{-4}\left|\Omega_{e}\right|$. The plasma parameters are mentioned in each panel.

and the FHFI regime is enlarged. These effects are boosted by increasing $\beta_{c}$, confirming the results in Figs. 5 and 6 . For the sake of comparison, in panel (b) we compare the FHFI and the WHFI thresholds for the same set of plasma parameters $\left(\delta=0.05, A_{c}=1.0\right)$, and different anisotropies $A_{b}=0.7,1.0,1.5$. As shown in panel (a), the unstable FHF modes require relatively high beaming velocities $u_{b}>2.7$ making the comparison relevant only for the WHFI upper threshold. For isotropic beams (red lines), the FHFI is dominant at low $\beta_{c}<0.55$ and high $u_{b}>2.7$, and also at beaming velocities exceeding the WHFI (upper) threshold, while the WHFI is dominant for less energetic beams with $u_{b}<2.7$. If $u_{b}>3.3$ and $\beta_{c}>0.55$ are high enough, we can identify a regime of transition (gray shaded regime) where the unstable FHF and WHF modes may co-exist and interplay. The anisotropic beams with $A_{b}=1.5$ determine the interplay regime to move towards higher $u_{b}>3.45$ and lower limit $\beta_{c}>0.49$. For an opposite anisotropy $A_{b}>0.7$, this regime moves towards lower $u_{b}>3.17$ and higher limit $\beta_{c}>0.78$. 
In order to complete the analysis, in Fig. 11, we describe the effect of the beam velocity on the thresholds of temperature anisotropy driven instabilities, WI in panel (a) and FI in panel (b). As expected, the WI threshold is markedly enhanced by the beam velocity $u_{b}=1.5$, confirming the inhibiting effect already shown on the growth-rates in Figs. 3 and 7. Here, we can see that this effect is reduced with increasing $\beta_{c}$. Also expected is the effect shown by the EFH threshold, which is markedly reduced in the presence of beam, see panel (b), confirming the stimulating effect of beams on the EFHI growth rates, obtained in Fig. 8.

\section{DISCUSSIONS AND CONCLUSIONS}

As shown in the introduction, the heat-flux instabilities may play a major role in the evolution of electron beams in the solar wind, but a definitive answer on these issues requires a detailed examination of these instabilities in conditions specific to space plasmas. The kinetic approach proposed in this paper enables an advanced characterization of the heat-flux instabilities for complex but realistic conditions, when the electron beams exhibit temperature anisotropies. The new unstable regimes uncovered here are controlled by two drivers, i.e., beaming velocity $u_{b}$ and beam anisotropy $A_{b}$, and by the core plasma beta $\beta_{c}$, and we have contrasted with idealized regimes of instabilities driven either by isotropic beams (Saeed et al., 2017a and Shaaban et al., 2018) or by non-drifting (core-beam) populations with temperature anisotropies.

For less energetic beams the WHFI is found to be very sensitive to the beam anisotropy: growth rates are markedly increased if $A_{b}>1.0$, and are decreased when $A_{b}<1.0$ (Fig. 1). Core anisotropy $A_{c} \neq 1.0$ shows similar effects on the WHFI, but it is much less effective than the beam anisotropy, see Fig. 2. The common WI can be excited at low $\beta_{c}$ $<1$ but for a significant $A_{b}>1.0$. The beam has an inhibiting effect on the WI: reducing growth rates and the range of unstable wave-numbers with increasing the beaming velocity. But, apparently, the beam may stimulate WI driven by the core anisotropy is $A_{c}>1.0$, see Figure. 3, where growth rates increase and saturate for higher beaming velocities, resembling a regime characteristic to WHFI.

Firehose instabilities are expected to develop for relatively higher $u_{b}$ or/and higher $\beta_{c}$, and contrary to whistlers, differences between FHFI and FI are easier to determine. FHFI can be excited even for a low $\beta_{c}<1$, provided the beam velocity is high enough, while FI requires a high $\beta_{c} \geqslant 3.0$ and a temperature anisotropy $A_{b}<1.0$. For a moderately high $\beta_{c}=1.2$, we have found that $A_{b}<1$ has a stimulating effect on the FHFI, increasing the growth rates and the corresponding wave frequencies (Fig. 5). The anisotropy in the perpendicular direction has an opposite effect, see Fig. 6 , where growth rates and wave frequencies of FHFI decrease with increasing $A_{b}>1$. These variations of the growth rates and wave frequencies with the temperature anisotropies have not been observed in the previous studies which were restricted to low $\beta_{c}=0.04$ regimes (Saeed et al., 2017b).
Figure 7 suggests that, depending on the beaming velocity $u_{b}$, the interplay with temperature anisotropy $A_{b}>1.0$ can be divided into two distinct regimes. For a beaming velocity below the threshold of FHFI, i.e., $u_{b}<2.8$, dominant is the WI, and, as expected, the beam has an inhibiting effect, reducing the growth rates and the range of unstable wave-numbers. In the second regime, more energetic beams with $u_{b} \geqslant 2.8$ excite the FHFI and the growth rates display a second distinct peak at low wave-numbers. The beam stimulates the FHFI, but inhibits the WI peak, and wave frequencies may change sign showing an LH polarization even in the range of the WI peak, under the influence of a dominant FHFI. Regarding the more common FHI driven by an excess of parallel temperature $(A<1)$, the effective free energy is enhanced in the presence of a beam, and FHI is stimulated (Fig. 8). Another remark can be made if we calculate the core drift velocity $u_{c}$ for the same plasma parameters used to derive the heat-flux unstable modes in Figs. 4(a) and 7(b). The plasma parameters used for the WHFI, e.g., $\delta=0.05$, $\beta_{c}=0.04$, and $u_{b}=U_{b} / c \omega_{e} /\left|\Omega_{e}\right|=0.6$, where $\omega_{e} /\left|\Omega_{e}\right|$ $=100$, imply for the core drift velocity $U_{c}=\delta U_{b}$ $(1-\delta)=3.16 \times 10^{-4}$, which is about 1.6 times higher than Alfvén velocity $V_{A}=2 \times 10^{-4} \mathrm{c}$, commonly invoked in similar studies. For the FHFI, we assumed $\delta=0.05, \beta_{c}=0.04$, and $u_{b}=3.8$, implying a higher core drift velocity $U_{c}$ $=10 V_{A}$. In the solar wind, $U_{c}$ is comparable to, or larger (three times larger in a collisionless plasma) than $V_{A}$ (Pulupa et al., 2014). Thus, our results strengthen the early predictions (Gary et al., 1975 and Gary and Li, 2000) that whistler instabilities could be more efficient in regulating the electron heat flux in the solar wind.

Thresholds displayed in Figs. 9-11 may provide a better overview on the interplay of these instabilities. In Fig. 9, the unstable WHF modes are located between two thresholds, namely, lower and upper thresholds. In terms of the beam velocity $u_{b}$, the interval of WHFI in between these two thresholds may significantly increase even for a modest temperature anisotropy in the perpendicular direction $A_{b}>1$, or it is markedly reduced by an opposite anisotropy in the parallel direction $A_{b}<1$. Situated above these thresholds, the unstable regime of the FHF modes, see Fig. 10(a), shows opposite effects, increasing when $A_{b}<1$ and diminishing for $A_{b}>1$. All these variations increase with increasing $\beta_{c}$. In Fig. 10(b), we have identified unstable regimes conditioned by both the WHFI and FHFI, which move either towards higher $u_{b}$ and lower $\beta_{c}$ if the anisotropy increases in the perpendicular direction $\left(A_{b}>1\right)$ or towards lower $u_{b}$ and higher $\beta_{c}$ by increasing the anisotropy in the parallel direction $\left(A_{b}<1.0\right)$. These unstable regimes are considerably enhanced by increasing $\beta_{c}$. In Fig. 11, we have described the effects of beam on the temperature anisotropy thresholds. The WI threshold is increased by increasing the beaming velocity, confirming the inhibiting effect on the growth rates in Figs. 3 and 7. On the other hand, the FI threshold is decreased by increasing the beaming velocity, confirming the stimulating effect on the growth rates in Fig. 8.

To conclude, we have identified new regimes of the whistler and firehose unstable modes, which are highly conditioned by the interplay of two sources of free energy, an 
electron beam and its intrinsic temperature anisotropy. This study is focused on parallel electromagnetic modes, with intention to facilitate the analysis and differentiate between different regimes of these instabilities. In the oblique directions, very efficient may be the aperiodic instabilities, like electron mirror or electron firehose, but their properties are known only for regimes triggered by the temperature anisotropies (Maneva et al., 2016 and Shaaban et al., 2018). Our results should therefore stimulate further investigations to address the full spectrum of beam-driven electromagnetic and electrostatic instabilities.

\section{ACKNOWLEDGMENTS}

The authors acknowledge support from the Katholieke Universiteit Leuven, Ruhr-University Bochum. These results were obtained in the framework of the project Nos. SCHL 201/35-1 (DFG-German Research Foundation), GOA/2015-014 (KU Leuven), G0A2316N (FWOVlaanderen), and C 90347 (ESA Prodex 9). S. M. Shaaban would like to acknowledge the support by a Postdoctoral Fellowship (Grant No. 12Z6218N) of the Research Foundation Flanders (FWO-Belgium) and the support by a Travel Grant for a long stay aboard (Grant No. V419818N) of FWO-Belgium. P.H.Y. acknowledges NSF Grant No. AGS1550566 to the University of Maryland, the Science Award Grant from the GFT Charity, Inc., to the University of Maryland, and the BK21 plus program from the National Research Foundation (NRF), Korea, to Kyung Hee University. Some basic ideas developed in this paper have been discussed at the 1st ISSI meeting of the international team: Kappa Distributions.

\section{APPENDIX: FITTING PARAMETERS IN EQ. (6)}

We have used Eq. (6) to describe the instability plasma conditions in terms of the instability thresholds from Figs. 9-11, defined by either the beam velocity or the temperature

TABLE II. Fitting parameters in Fig. 9.

\begin{tabular}{lccccccc}
\hline \hline & \multicolumn{3}{c}{ WHFI (a) } & & \multicolumn{3}{c}{ WHFI (b) } \\
\cline { 2 - 4 } \cline { 6 - 8 }$A_{b}$ & 0.9 & 1.0 & 1.1 & & 0.9 & 1.0 & 1.1 \\
\hline$a$ & 0.13 & 0.03 & -1.32 & & -0.023 & -0.04 & -0.01 \\
$b$ & 1.0 & 1.0 & -0.29 & & 1.0 & 1.0 & 1.0 \\
$c$ & 0.30 & 0.26 & 0.0 & & 4.72 & 4.67 & 4.81 \\
$d$ & -0.22 & 0.18 & 1.0 & & -0.52 & -0.54 & -0.53 \\
\hline \hline
\end{tabular}

TABLE III. Fitting parameters in Fig. 10.

\begin{tabular}{lccccccc}
\hline \hline & \multicolumn{3}{c}{ FHFI } & & \multicolumn{3}{c}{ WHFI } \\
\cline { 2 - 4 } \cline { 6 - 8 }$A_{b}$ & 0.7 & 1.0 & 1.5 & & 0.7 & 1.0 & 1.5 \\
\hline$a$ & -0.003 & 2.45 & 2.72 & & 5.82 & -0.023 & -0.008 \\
$b$ & 1.0 & -0.1 & -0.14 & & 1.0 & 1.0 & 1.0 \\
$c$ & 3.21 & 0.0 & 0.0 & & 0.55 & 4.72 & 5.11 \\
$d$ & -0.03 & 1.0 & 1.0 & & -1.54 & -0.52 & -0.53 \\
\hline \hline
\end{tabular}

TABLE IV. Fitting parameters in Fig. 11.

\begin{tabular}{lccccc}
\hline \hline & \multicolumn{2}{c}{ WI } & & \multicolumn{2}{c}{ FI } \\
\cline { 2 - 3 } \cline { 5 - 6 }$u_{b}$ & 0.0 & 1.5 & & 0.0 & 2.5 \\
\hline$a$ & 0.012 & 0.018 & & -4.0 & -1.34 \\
$b$ & 1.0 & 1.0 & & 1.0 & 1.0 \\
$c$ & 1.05 & 1.32 & & 0.80 & 0.85 \\
$d$ & 0.017 & 0.072 & & -0.004 & 0.006 \\
\hline \hline
\end{tabular}

anisotropy (two distinct drivers), as a function of the core plasma beta. The fitting parameters $a, b, c$, and $d$ are tabulated in Tables II and III for the heat flux instabilities (WHFI and FHFI), and in Table IV for the anisotropy driven instabilities (WI and FI).

Breneman, A., Cattell, C., Wygant, J., Kersten, K., Wilson, L. B., Schreiner, S., Kellogg, P. J., and Goetz, K., "Large-amplitude transmitter-associated and lightning-associated whistler waves in the Earths inner plasmasphere at L $<2$," J. Geophys. Res.: Space Phys. 116(6), 1-10, https://dx.doi.org/ 10.1029/2010JA016288 (2011).

Fried, B. and Conte, S., The Plasma Dispersion Function (Academic Press, New York, 1961).

Gary, S. P., "Electromagnetic electron beam instabilities: Hot, isotropic beams," J. Geophys. Res. 90(A11), 10815, https://doi.org/10.1029/ JA090iA11p10815 (1985).

Gary, S. P., Theory of Space Plasma Microinstabilities (Cambridge University Press, 1993).

Gary, S. P., Feldman, W. C., Forslund, D. W., and Montgomery, M. D., "Heat flux instabilities in the solar wind," J. Geophys. Res. 80(31), 4197-4203, https://doi.org/10.1029/JA080i031p04197 (1975).

Gary, S. P. and Li, H., "Whistler heat flux instability at high beta," Astrophys. J. 529(2), 1131-1135 (2000).

Gary, S. P. and Saito, S., "Broadening of solar wind strahl pitch-angles by the electron/electron instability: Particle-in-cell simulations," Geophys. Res. Lett. 34(14), L14111, https://doi.org/10.1029/2007GL030039 (2007).

Gary, S. P. and Wang, J., "Whistler instability: Electron anisotropy upper bound,” J. Geophys. Res.: Space Phys. 101(A5), 10749-10754, https:// doi.org/10.1029/96JA00323 (1996).

Lacombe, C., Alexandrova, O., Matteini, L., Santolík, O., CornilleauWehrlin, N., Mangeney, A., de Conchy, Y., and Maksimovic, M., "Whistler mode waves and the electron heat flux in the solar wind: Cluster observations," Astrophys. J. 796(1), 5 (2014).

Lazar, M., Shaaban, S. M., Fichtner, H., and Poedts, S., "Temperature anisotropy instabilities stimulated by the interplay of the core and halo electrons in space plasmas," Phys. Plasmas 25(2), 022902 (2018).

Lengyel-Frey, D., Hess, R., MacDowall, R., Stone, R., Lin, N., Balogh, A., and Forsyth, R., "Ulysses observations of whistler waves at interplanetary shocks and in the solar wind," J. Geophys. Res.: Space Phys. 101(A12), 27555-27564, https://doi.org/10.1029/96JA00548 (1996).

Lin, N., Kellogg, P., MacDowall, R., Scime, E., Balogh, A., Forsyth, R., McComas, D., and Phillips, J., "Very low frequency waves in the heliosphere: Ulysses observations,” J. Geophys. Res.: Space Phys. 103(A6), 12023-12035, https://doi.org/10.1029/98JA00764 (1998).

Maksimovic, M., Zouganelis, I., Chaufray, J.-Y., Issautier, K., Scime, E., Littleton, J., Marsch, E., McComas, D., Salem, C., Lin, R. et al., "Radial evolution of the electron distribution functions in the fast solar wind between 0.3 and 1.5 au," J. Geophys. Res.: Space Phys. 110(A9), A09104, https://dx.doi.org/10.1029/2005JA011119 (2005).

Maneva, Y., Lazar, M., Vinas, A., and Poedts, S., "Mixing the solar wind proton and electron scales: Effects of electron temperature anisotropy on the oblique proton firehose instability," Astrophys. J. 832(1), 64 (2016).

Paesold, G. and Benz, A. O., "Electron firehose instability and acceleration of electrons in solar flares," Astron. Astrophys. 351, 741-746 (1999).

Pagel, C., Gary, S. P., de Koning, C. A., Skoug, R. M., and Steinberg, J. T., "Scattering of suprathermal electrons in the solar wind: ACE observations," J. Geophys. Res. (Space Phys.) 112, A04103, https://dx.doi.org/ 10.1029/2006JA011967 (2007). 
Phillips, J., Gosling, J., McComas, D., Bame, S., Gary, S., and Smith, E.,, "Anisotropic thermal electron distributions in the solar wind," J. Geophys. Res.: Space Phys. 94(A6), 6563-6579, https://doi.org/10.1029/ JA094iA06p06563 (1989).

Pierrard, V., Lazar, M., Poedts, S., Štverák, Š., Maksimovic, M., and Trávníček, P., "The electron temperature and anisotropy in the solar wind. Comparison of the core and halo populations," Sol. Phys. 291(7), 2165-2179 (2016).

Pilipp, W., Miggenrieder, H., Montgomery, M., Mühlhäuser, K.-H., Rosenbauer, H., and Schwenn, R., "Characteristics of electron velocity distribution functions in the solar wind derived from the helios plasma experiment," J. Geophys. Res.: Space Phys. 92(A2), 1075-1092, https:// doi.org/10.1029/JA092iA02p01075 (1987).

Pulupa, M. P., Bale, S. D., Salem, C., and Horaites, K., "Spin modulated spacecraft floating potential: Observations and effects on electron moments," J. Geophys. Res.: Space Phys. 119(2), 647-657, https://doi.org/ 10.1002/2013JA019359 (2014).

Saeed, S., Sarfraz, M., Yoon, P. H., Lazar, M., and Qureshi, M. N. S., "Electron heat flux instability," Mon. Not. R. Astron. Soc. 465(2), 1672-1681 (2017a).

Saeed, S., Sarfraz, M., Yoon, P. H., and Qureshi, M. N. S., "Characteristics of heat flux and electromagnetic electron-cyclotron instabilities driven by solar wind electrons," Mon. Not. R. Astron. Soc. 4936, stx049 (2017b).

Saito, S. and Gary, S. P., "Whistler scattering of suprathermal electrons in the solar wind: Particle-in-cell simulations," J. Geophys. Res. (Space Phys.) 112, A06116, https://dx.doi.org/10.1029/2006JA012216 (2007).
Shaaban, S., Lazar, M., Astfalk, P., and Poedts, S., "Stimulated mirror instability from the interplay of anisotropic protons and electrons, and their suprathermal populations," J. Geophys. Res.: Space Phys. 123(3), 1754-1766, https://doi.org/10.1002/2017JA025066 (2018).

Shaaban, S., Lazar, M., Poedts, S., and Elhanbaly, A., "The interplay of the solar wind proton core and halo populations: Emic instability," J. Geophys. Res.: Space Phys. 121(7), 6031-6047, https://doi.org/10.1002/ 2016JA022587 (2016).

Shaaban, S. M., Lazar, M., and Poedts, S., "Clarifying the solar wind heatflux instabilities," Mon. Not. R. Astron. Soc. 2018, sty 1567.

Štverák, Š., Maksimovic, M., Trávníček, P. M., Marsch, E., Fazakerley, A. N., and Scime, E. E., "Radial evolution of nonthermal electron populations in the low-latitude solar wind: Helios, cluster, and ulysses observations," J. Geophys. Res. 114, A05104, https://doi.org/10.1029/2008JA013883 (2009).

Štverák, Š., Trávníček, P., Maksimovic, M., Marsch, E., Fazakerley, A. N., and Scime, E. E., "Electron temperature anisotropy constraints in the solar wind," J. Geophys. Res. 113, A03103, https://doi.org/10.1029/ 2007JA012733 (2008).

Viñas, A., Gurgiolo, C., Nieves-Chinchilla, T., Gary, S., and Goldstein, M., "Whistler waves driven by anisotropic strahl velocity distributions: Cluster observations," AIP Conf. Proc. 1216, 265-270 (2010).

Vocks, C., Salem, C., Lin, R. P., and Mann, G., "Electron halo and strahl formation in the solar wind by resonant interaction with whistler waves," Astrophys. J. 627, 540-549 (2005). 